\title{
Endocrine treatment of prostate cancer - recent developments and the future. Part 1: maximal androgen blockade, early vs delayed endocrine treatment and side-effects
}

\author{
F.H. SCHRÖDER \\ Department of Urology, Erasmus University and Academic Hospital Rotterdam, The Netherlands
}

\section{Introduction}

The endocrine treatment of prostate cancer has changed rapidly in the last few years, a change made possible and necessary because of several important developments. Maximal androgen blockade (MAB), which has occupied clinical research and resources for more than 10 years, has found a modest but clearly defined place in the armamentarium of the endocrine management of metastatic prostate cancer. This development arrests research potential, eliminates the need to use MAB as the 'gold standard' in metastatic and nonmetastatic disease and allows concentration on other relevant issues which have not been resolved, but which are of great importance for the further development of the field. Such unresolved issues are the question of early vs delayed treatment, the value of adjuvant endocrine treatment, the evaluation of intermittent treatment, the possibility of using very early endocrine treatment, aspects related to endocrine prevention, and the possibility of preserving quality of life, especially as it is related to sexual performance for at least some period.

Another important aspect is not related to treatment, but to the disease. Endocrine treatment is bound to change because prostate cancer is diagnosed much earlier in life and with more favourable disease characteristics. Prostate cancer is more frequently treated by potentially curative means, but up to $30-40 \%$ of such patients may escape such treatment and will eventually require early or delayed endocrine treatment. The duration of endocrine treatment in patients who present with signs of biochemical progression after radiotherapy or radical prostatectomy likely to be very much longer than ever experienced before. How can we cope with this situation without imposing unacceptable side-effects, which are related to such long durations of endocrine treatment? Which aspects of endocrine treatment need to be studied to provide future forms of endocrine management that are adapted to individual clinical situations, the need to prevent long-term side-effects, e.g. osteoporosis, anaemia, and muscle wasting, the necessity to provide an optimal quality of life, especially during the early phase of endocrine treatment and, if possible, with preservation of sexual function?

The present author summarized the issue of the endocrine treatment of prostate cancer in the recent edition of Campbell's Urology [1]. That chapter was written in 1996 and many aspects are now outdated; the present review attempts to provide an update, to present a view on future research necessities and to anticipate clinical developments.

\section{Maximal androgen blockade}

$\mathrm{MAB}$ as a concept of treatment for prostate cancer is the simultaneous complete elimination or blockade of testicular and adrenal androgens. In the literature, the synonyms complete androgen withdrawal, complete androgen blockade and total androgen blockade are used. The concept was first suggested by investigators from Italy and Germany in the 1960s and 1970s. The first randomized controlled study which tested MAB was the EORTC Genitourinary (GU) Group protocol 30805. This protocol was developed in 1979 and compared $\mathrm{MAB}$ achieved by castration plus cyproterone acetate (CPA) $150 \mathrm{mg} /$ day to castration as a standard treatment, and to DES $1 \mathrm{mg}$ in the third arm; 335 patients with metastatic disease were recruited. The final analysis [2] showed no differences in time to progression and overall survival among the three treatment arms. In 1983, based on experimental findings, Labrie et al. [3] began to popularize the concept on the basis of a very small phase II study. Subsequently, many randomized comparative studies using various forms of MAB have been carried out in the hope that MAB would significantly delay the progression to endocrine independence of prostate cancer, and eventually prolong survival. Among more than 20 studies that address the issue, there were two major protocols that seemed to fulfil these hopes, and to establish $\mathrm{MAB}$ as the standard treatment for metastatic prostate cancer [4,5]. However, methodological problems around the largest of these studies, protocol 
0036 of the South West Oncology Group (SWOG)/ Intergroup [4] cast doubt on the validity of these data early on, mainly because the LHRH agonist in the control arm was used without antiandrogen protection in the initial phase of treatment, and differences in time to progression suggested an effect of 'flare' in the control arm. The SWOG/Intergroup decided subsequently to carry out another study and to replace the LHRH arm by castration, as had been done in the similar protocol of the EORTC GU Group [5]. The results of this latter protocol (SWOG/Intergroup Study 105) together with the results of a comprehensive meta-analysis have been decisive in judging the true value of MAB. The data resulting from these two studies will now be reviewed.

\section{The meta-analysis}

Why choose a meta-analysis, when a randomized study should be the most valid way of testing treatment principles? Apparently this is not so; if only small or no differences exist between treatment regimens, a very large sample is necessary. Clinical trials, even if randomized, usually do not have the necessary power. In this situation, there is a some chance that even valid trials with the same methodology can lead to variable results. The chance of finding a positive or negative result in a situation where in fact no difference exists is estimated to be $\approx 10 \%$. This error can be avoided by a meta-analysis. Another reason to use meta-analyses is the possibility of avoiding publication bias and the bias resulting from not considering papers written in languages that are difficult to access. This has been called the 'Tower of Babel' bias. Publication bias results from the reluctance of study groups, authors and publishers to present and accept negative results for publication. A meta-analysis or overview analysis must include all studies, including those that are unpublished. A metaanalysis must access the original data, as the use of published data alone is unacceptable. Because a metaanalysis often has to disregard details which are addressed in individual trials, such as important prognostic factors, the meta-analysis has been blamed for "comparing apples and pears'. However, these pitfalls can be avoided by running multiple analyses in which patients are separated according to such factors. Another prerequisite for conducting a meta-analysis is that all trials conducted before a fixed date are included. The relative value of large randomized trials and meta-analysis has recently been discussed in depth by LeLorier et al. [6].

Almost all trials using MAB have been included in a meta-analysis conducted by the Prostate Cancer Trialists Cooperative Group (PCTCG) [7]. This study included 22 of 25 randomized studies of various regimens of MAB, which were initiated before December 1989. The analysis included the individual data of 5710 patients, of whom 3283 had died. Another description of the meta-analysis, which is in many respects complementary, was given by Dalesio et al. [8]. Unfortunately, the data from three suitable trials could not be obtained. The endpoint of the analysis was the 5-year overall survival; separate analyses for each treatment regimen were also carried out, and a common analysis of all trials together. Of all patients, $13 \%$ had nonmetastatic disease; a separate analysis of those with metastatic disease with and without M0 cases did not alter the final results. While there were differences in 5-year survival, mainly related to the use of three different antiandrogens (flutamide, nilutamide and CPA), in none of these subgroup analyses was there a significant improvement in 5-year survival; Fig. 1. shows the survival estimates for all 5710 patients. There was an advantage for MAB at 5 years of $3.5 \%$, which was not statistically significant. This metaanalysis is currently being updated; the total number of patients is now $\approx 7500$, with $\approx 5200$ deaths.

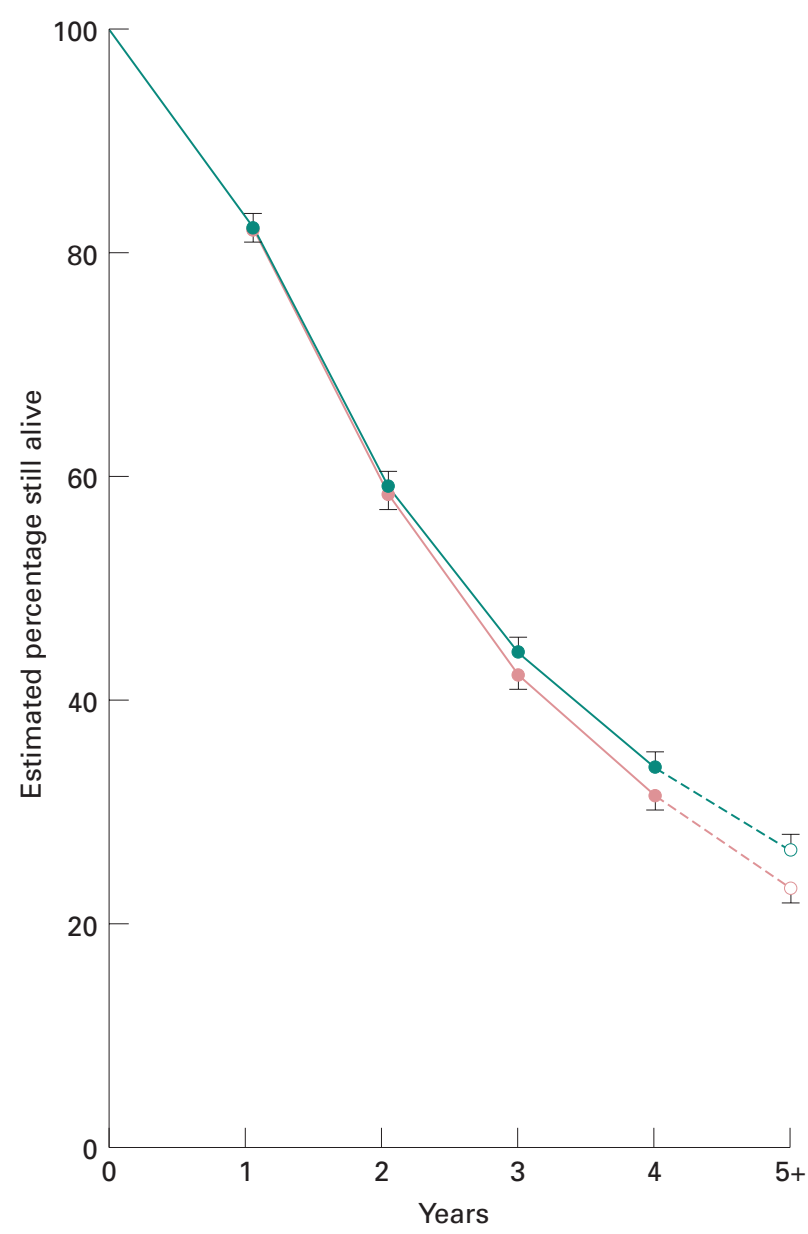

Fig. 1. Survival in 22 randomized prostate cancer trials of $\mathrm{MAB}+$ castration (green) vs castration alone (red); 5710 patients, 3283 deaths (from [7], with permission). 
Considering the negative outcome of the studies that have been added, it cannot be expected that there will be a larger difference in favour of MAB. However, considering the increase in power, a difference of $\approx 3 \%$ may be statistically significant. The question will then be; should every patient with metastatic prostate cancer receive $\mathrm{MAB}$, to produce a $3.5 \%$ advantage in 5-year survival? Side-effects, other therapeutic options, quality of life and cost will have to be considered in this context.

\section{SWOG/Intergroup study 105}

Recently, the results of this important protocol have been published [9]; the study compares bilateral orchidectomy combined with flutamide vs placebo in patients with metastatic prostate cancer. The study is doubleblind for flutamide and placebo; 1387 patients were randomized between 15 December 1989 and 15 September 1994. At the time of the analysis presented in 1997, the study was mature. The main endpoints are survival and progression-free survival. A stratification by minimal disease (MD, 283 patients) and extensive disease (ED, 1088 patients) was carried out to confirm differences found in the earlier protocol [4], in which MAB seemed to be more effective in patients with MD. The follow-up was 3 monthly and the results of protocol 105 , as they were presented in 1997, are reproduced in Table 1. Of 1387 patients, 1371 were eligible; more than half of all patients have progressed and/or died. Toxicity favoured the placebo group, with significant differences for diarrhoea $(P<0.001)$ and anaemia $(P=$ $0.046)$ and a trend toward more favourable liver function. The results are given for the treatment groups of orchidectomy plus flutamide (OF) and orchidectomy plus placebo (OP); similar numbers of patients were eligible for evaluation. Also, the proportions of patients with MD and ED were similar in the two treatment groups. There were no significant differences in median or overall survival.

An important question in the further exploration of endocrine treatment is whether PSA could be used as a surrogate marker. This would allow a shortcut in the trial design by avoiding the necessity of waiting for progression to hormone-independence and death from prostate cancer. If a decrease of PSA to $<4.0 \mathrm{ng} / \mathrm{mL}$ was considered as a response, there was a significant advantage in the proportion of responses in favour of the flutamide group (OF $80 \%$, OP $68 \%, P=0.001$ ). Clearly, this is not reflected in an advantage for the main endpoints of the trial. In this trial, PSA cannot be considered a surrogate endpoint.

Reviewing the results of this largest single, wellcontrolled study together with the results of the metaanalysis, MAB is clearly not the treatment of choice for metastatic prostate cancer. It is possible that subgroups of patients would benefit from MAB, but these have not been properly identified.

\section{Remaining indications for $M A B$}

If endocrine treatment by the use of an LHRH agonist is chosen, an antiandrogen should be added before the initiation of, or at the time of treatment to suppress disease 'flare'. Many trials that have compared MAB to more standard forms of endocrine treatment, and which have studied response as a study endpoint, have found that tumour markers become normal and symptoms of prostate cancer resolve more quickly, and often significantly more frequently (as in SWOG/Int 105), than with castration or the use of LHRH agonists. The use of MAB, at least temporarily, should be considered in patients who initially present with symptoms related to prostate cancer (e.g. bone pain, neurological symptoms, impaired micturition). A faster and more pronounced response can be expected; however, this may not be expected to translate into an advantage in time to progression and/or survival.

The role of second-line endocrine treatment with antiandrogens needs to be further investigated; available information is old and poorly controlled. Further research to identify subgroups that may benefit from MAB may be warranted. However, without repeating very large studies, this may prove to be impossible.
Table 1 The SWOG/INT 105 trial, a randomized study of metastatic prostate cancer comparing castration and placebo versus castration and flutamide in a double-blind protocol (after [9])

\begin{tabular}{llllll}
\hline & & & & \multicolumn{2}{l}{ Median times (months) } \\
\cline { 5 - 6 } Group* & Eligible & MD* & $E D^{*}$ & $\begin{array}{l}P F^{*} \\
\text { all/MD }\end{array}$ & $\begin{array}{l}\text { Survival } \\
\text { all/MD }\end{array}$ \\
\hline OF & 690 & 139 & 551 & $20 / 50$ & $32 / 54$ \\
OP & 681 & 144 & 537 & $19 / 47$ & $30 / 51$ \\
\hline
\end{tabular}

${ }^{*} \mathrm{OF}$, orchidectomy + flutamide; OP, orchidectomy + placebo; MD, minimal disease; ED, extensive disease; PF, progression-free. 


\section{Early vs delayed endocrine treatment}

\section{The scope of the problem}

The issue of early vs delayed treatment used to be debated mainly for two clinical situations; (i) should locally advanced but nonmetastatic disease be treated immediately, or once metastatic progression had occurred?; and (ii) should patients with lymph node metastases be treated immediately after diagnosis, or whenever metastatic progression occurs?

Recently, the scope of the problem has become much wider. Evidence for tumour progression is no longer based on an elevation of acid phosphatase, alkaline phosphatase, positive bone scans, metastases visible on X-rays and complaints of patients related to various types of progression, including local progression. Local progression after potentially curative management and metastatic progression of any type are heralded long before metastases can be diagnosed by a rise in the serum level of PSA. The availability of PSA as a marker of 'biochemical progression' has advanced by some years the possibility of identifying tumour progression and, if this is considered an indication for treatment, also increased the time during which endocrine treatment might be applied. Figure 2 shows an attempt to quantify this changing situation. Estimates of median times to clinical progression and

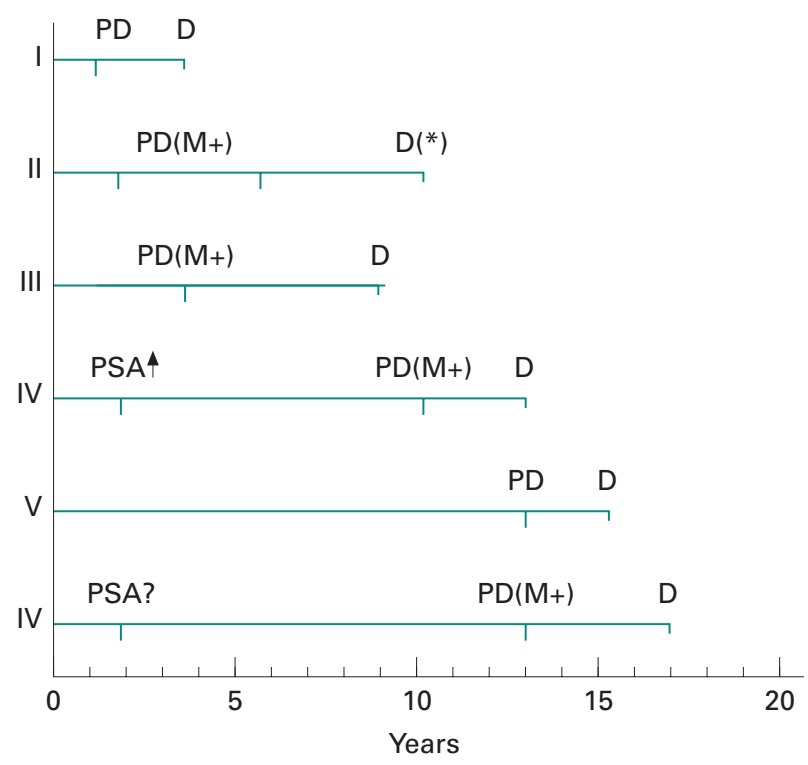

Fig. 2. The estimated median times to biochemical and clinical progression, and to death for each stage of prostate cancer at diagnosis. (PD, progressive disease; D, death). *, Median times with delayed treatment and early treatment are around 20 and 60 months. I, $\mathrm{M}+[2,11]$. II, $\mathrm{N}+\mathrm{MO}[12] \mathrm{PD}$ on delayed treatment, time of I added. III, T3MO[13,10]. IV, T2 progression after radical prostatectomy $[15,16]$. V, T2(T1c)[17,18] (after watchful waiting) disease specific. VI, T1a[19,20,21]. death are used for each stage of the disease, taken from the available literature on the natural history and from clinical trials of prostate cancer. In Fig. 2-I, the clinical course of M1 disease is depicted; the median survival rates for $\mathrm{M}+$ disease are extrapolated from two EORTC protocols $[2,11]$, which used MAB and standard treatment without showing a difference; the data given in [7] and [9] are similar. Half of the patients with metastatic prostate cancer are dead within 30-36 months. To this period of 3 years from the diagnosis of metastatic disease to death, one year was added to account for the earlier diagnosis of $\mathrm{M}+$ disease during follow-up. A 4-year period from the diagnosis of M1 to death was included in all time estimates in Fig. 2-II-IV. Fig. 2-II, which relates to $\mathrm{N}+\mathrm{M} 0$ disease, shows a drastically longer time from diagnosis to metastatic progression. In a sample of 34 cases from a randomized prospective study of $\mathrm{N}+\mathrm{M} 0$ cases (EORTC protocol 30846) the median time to progression in 34 treated patients was 6 years [12]; T3 prostate cancer seems to be quite similar to $\mathrm{N}+\mathrm{M} 0$ disease, but only a few observations on the natural history are available. From the placebo groups to which delayed treatment was applied within the Veterans Administrative Cooperative Urological Group (VACURG) studies 1 and 2 [13], median times of almost 7 years (study 1 ) and 4 years (study 2) can be extrapolated for progression from stage 3 to stage 4 . The mean time to progression to metastatic disease of nonmetastatic patients in the MRC study (MRC Prostate Cancer Working Party, [10]) was 27 months. Adolfsson et al. [14] found a median time to progression in T3 G1 + 2 disease of about 7 years. A 4 -year period is inserted for progression to $\mathrm{M}+$ disease in Fig. 2-III, relating to $\mathrm{T} 3$ disease. These differences show how relative and inaccurate such historical comparisons may be. The assumption made for $\mathrm{N}+$ and $\mathrm{T} 3$ disease, that the time from metastatic progression to death is comparable to cases presenting with metastases at diagnosis, may also be very inaccurate. Within a follow-up scheme, metastatic disease may be detected much earlier and therefore this period may be longer. Obviously, the time of overall survival is also influenced by age at diagnosis. However, to make the point of this discussion, exact estimates are unnecessary. Figure 2-IV is based on solid information and relates to men who presented with apparently locally confined disease (T2), who underwent radical prostatectomy, and subsequently suffered progression. Median times from radical prostatectomy to PSA progression, and from there to metastatic progression and death, were recently reported based on the data from the Johns Hopkins Institute. The median time from PSA progression to metastatic progression in this series was $\approx 8$ years, but depended on prognostic factors such as grade [15]; in the Rotterdam radical prostatectomy series, the respective value was 6 years [16]. These data show that locally 
confined disease, even if it progresses after radical prostatectomy, on average has a slow course and may be associated with a median duration of untreated and treated natural history of about 12-13 years. Similar projections can be made using the data of Johansson et al. [17] and Adolfsson et al. [18], but have the disadvantage of excluding grade 3 disease. Figure 2-VI depicts the natural course of truly focal disease (T1a), as it is identified as an incidental finding in benign prostatic specimens removed for symptomatic BPH. Only one report gives a median time to progression, of $\approx 13.5$ years for A1 disease in 143 patients [19]. This median time can be extrapolated from Kaplan-Meier projections. Epstein et al. [20] reported $16 \%$ progression with 96 months of follow-up, and Blute et al. [21] $26.6 \%$ with 10.2 years of follow-up. It is estimated that the median time to clinical progression may be 13-15 years. The lead-time, i.e. the time by which the diagnosis is advanced by the use of early detection regimens, e.g. achievable using PSA-driven screening, may be $4-10$ years [22,23]. In future, with increasing screening for prostate cancer, this amount of time may have to be added to the clinical course of many patients. This also means that there will be a shift from more extensive to less extensive disease at the time of diagnosis. This trend is confirmed by many recent studies in the USA, and on the European continent [24,25].

How does all this relate to the question of early vs delayed endocrine treatment? Obviously, if for example in Fig. 2-IV, the situation of PSA progression after radical prostatectomy (or radiotherapy), endocrine treatment is given at the time of PSA elevation, the duration of endocrine treatment will be drastically increased. This duration is unknown at present. However, the median time to metastatic progression without endocrine treatment is 6-8 years. Endocrine treatment periods of 10 years in this and similar situations must be anticipated. Also, the application of this treatment will increase as more patients will be diagnosed with locally confined disease in the future, and will receive potentially curative treatment. Obviously, the side-effects of endocrine treatment over very long periods will differ importantly from those that are experienced in patients who present with locally advanced or metastatic disease, because of the much longer duration of treatment. This issue will be dealt with later in this article.

In this context, what is early endocrine treatment? Is it treatment given at the time of diagnosis of metastatic disease, of lymph node positive disease, at the time of PSA progression, or at the time of diagnosis of any type of prostate cancer? If we examine the issue of early vs delayed endocrine treatment in metastatic disease, locally extensive disease, or in lymph node positive disease, are the results obtained applicable to situations where signs of progression are detected much earlier in the clinical course, or even to prostate cancer just diagnosed in locally confined stages? There are currently no answers to these questions. However, the clinician who wishes to apply early treatment in very early stages of the disease needs to be aware of the natural course for each stage, as it is roughly indicated in Fig. 2. Are we doing more harm than good if we treat patients for periods of $\geqslant 10$ years by endocrine means? It will be shown below that the expected side-effects will have to be matched by a significant prolongation of life for this to be acceptable. Meanwhile, the available information arising from randomized studies of early vs delayed endocrine treatment remain to be reviewed.

\section{The VACURG studies}

The first study that addressed this question, albeit indirectly, was VACURG study 1, a randomized study of patients with locally extensive and metastatic prostate cancer, comparing $5 \mathrm{mg}$ DES with castration, to castration $+5 \mathrm{mg}$ DES, and to placebo $[13,26]$. In this study, when progression to symptomatic metastatic disease occurred, $44 \%$ of the patients randomized to placebo transferred to active endocrine treatment. Because there was no difference in overall survival, comparing the placebo group with the active treatment groups, one of the conclusions of the study was that it did not matter whether treatment was instituted early or later during the clinical course. VACURG study 2, which included low dosages of DES $(0.2$ and $1.0 \mathrm{mg})$, was set up to exclude the cardiovascular toxicity seen in study 1 with $5 \mathrm{mg}$ DES. Study 2 was discontinued because again, in the $5 \mathrm{mg}$ DES arm of the study, there was an excess of cardiovascular death. With only 506 patients randomized (475 were randomized to each of the four treatment groups in study 1), the power of this study was limited. However, the patients treated with $1 \mathrm{mg}$ DES showed a significant advantage in overall survival over the placebo group and the other DES groups. This study was extensively analysed and as a result the advice about early vs delayed endocrine treatment resulting from study 1 was revised. The final conclusion was that younger patients with poorly differentiated tumours should be treated immediately, and that older patients and patients with low-grade disease (Gleason score 2-6) probably do not need early hormone therapy [13].

\section{The MRC trial}

The conclusions from VACURG Study 2 have not found widespread acceptance. However, patients and treating urologists are reluctant to see tumours progress with rising PSA levels and without treatment. It is probably mainly for this reason that early endocrine treatment is 
used increasingly often. The only truly randomized study addressing the issue has recently been published by the MRC Prostate Cancer Working Party Investigators Group [10] (subsequently referred to as the MRC study). The study was designed to compare the effect of early endocrine treatment vs delayed endocrine treatment in patients with locally extensive or asymptomatic metastatic prostate cancer, which was previously untreated. A total of 934 patients were randomized, 469 to immediate and 465 to deferred treatment (503 M0, $261 \mathrm{M} 1$ and $174 \mathrm{Mx}$ ). Of the patients who died, $67 \%$ did so from prostate cancer. There was a significant difference in favour of immediate treatment for overall survival of all patients $(P=0.02)$. There were larger and statistically significant differences for time to death from any cause in MO patients, as well as time to death from prostate cancer in M0 and M1 patients; 203 patients died from prostate cancer in the immediate and 257 in the delayed treatment arm, a difference of 54 cancer deaths, which explains all significant differences encountered, as time to death from causes other than prostate cancer did not differ between the treatment groups. The authors report that of the 54 cases who died in the delayed treatment arm, 29 (54\%) never received endocrine treatment. The participants in this protocol were encouraged to manage their patients 'according to their clinical practice'; a follow-up form was requested once a year. Bone scans were not available for many of the participants. If progressive disease had been diagnosed with a 3-monthly follow-up and treated appropriately, how would that have changed the final outcome? Is it inevitable to miss progression in some patients? Ongoing EORTC studies on the same issue do not confirm this possibility.

\section{What to do?}

Other studies, mainly at the level of the EORTC GU Group, are currently underway. Results based on at least 1500 patients in two protocols comparing early vs delayed treatment in lymph node-positive disease (EORTC 30846), and in M0 cases with all local stages (EORTC protocol 30891) will be ready for final evaluation in the near future. Preliminary evaluation of these protocols has shown that only very few patients will ever progress and die from prostate cancer without receiving endocrine treatment. Also, the serious complications (spontaneous fractures, paraplegia, etc.) described in the MRC study are rare in these studies. Clearly, a patient with locally advanced or metastatic prostate cancer, in whom endocrine treatment is delayed, has to have the opportunity and must be ready to undergo regular follow-up examinations, probably at 3-monthly intervals.

In the author's view, conclusive data which dictate either the use of early or delayed treatment are currently unavailable. Also, it is very unclear whether the preliminary information that is available can be applied to the situation of very early progression, such as a minimal rise in PSA level after radical prostatectomy or radiotherapy, or whether the question of early vs delayed treatment will find different answers by disease stage. The treating urologist and the patient have to be aware of the long duration of endocrine treatment, if it is applied early, and of the accumulation of side-effects that may occur with prolonged treatment.

What are the potential benefits and pitfalls of early vs delayed endocrine treatment? Take the example of a patient with an intact libido and who is sexually active in some way, not necessarily with intact erections; he is 65 years old and has a life expectancy without prostate cancer of $\approx 15$ years. He has a rising PSA level after radical prostatectomy or radiotherapy. What does early treatment offer? As detailed above, the median time to metastatic progression in this case is 6-8 years and depends on the original tumour characteristics. It is well known and understood that endocrine treatment prolongs time to progression. Treatment with a $5 \alpha$-reductase inhibitor in this situation will delay the rise of PSA and of progression by 9 months [27]. It has been shown that more aggressive endocrine treatment will then decrease the PSA level and will again produce a period of PSAbased remission of probably several years, say three. This means that a patient who chooses early endocrine treatment in this situation is likely to be treated for 9-12 years until he experiences metastatic progression. If that occurs, there will be no effective treatment in reserve. In the meantime, he has lost libido and potency, and has encountered several side-effects, elaborated below. In the worst case, he has suffered a hip fracture or other spontaneous fracture due to osteoporosis, which could have been avoided otherwise. At the time of metastatic progression, unfortunately no effective therapeutic options are available and the patient will then probably succumb within a year.

What does delayed treatment offer? The patient will have to live with the knowledge of a rising PSA level. If this keeps him awake at night, he is symptomatic and must be treated. In my experience of this situation, explanations such as mentioning the well-known median times until metastatic disease occurs help the patients to understand better what they may and may not expect. Also, to indicate that metastatic disease is rarely seen with PSA values of $<100-200 \mathrm{ng} / \mathrm{mL}$ gives them an idea of how relative a PSA increase from, e.g. 2 to $4 \mathrm{ng} / \mathrm{mL}$, may be. Meanwhile, during watchful waiting the patient can enjoy a normal libido and sexual life. He is not at risk from the side-effects of endocrine treatment and, if progression finally occurs, an effective treatment will be in reserve. 
In the author's view, both options should be explained to patients and they should be given a choice, as long as clear advantages for disease-specific and overall survival have not been shown. The EORTC GU Group protocol 30846, of which the randomization scheme is shown in Fig. 3, has been in progress since 1984. About 290 patients have been included. Despite very large differences in time to progression in the two arms, and despite two reviews by the Data Monitoring Committee that functions as an ethical supervisory body of this study, the protocol has not been discontinued because apparently there have been no differences in survival; the situation around protocol 30891 is similar.

\section{Side-effects of endocrine treatment}

Endocrine treatment leads to loss of libido and potency; it is thought that after castration this effect is immediate and it seems to be related to the elimination of testicular androgens. However, in a classic paper, Ellis et al. [28] found that after castration, treatment with oestrogens or the combination of both regimens, 16 of 38 previously potent patients remained potent for prolonged periods. This finding is confirmed by recent data relating to the use of antiandrogen monotherapy [29]. These data are derived from EORTC GU Group protocol 30892, a randomized study of patients with metastatic prostate cancer treated by either flutamide or CPA monotherapy. Figure 4 shows the time to loss of ejaculation in 75 previously potent patients. The Kaplan-Meier projections of loss of other sexual functions are reported elsewhere [29]. The important message is that the loss of sexual function under monotherapy with either a pure or a steroidal antiandrogen is a slow process. It does not seem to be true that sexual function is preserved with flutamide monotherapy, as has been claimed previously. However, $10-20 \%$ of patients remain potent over observation times of 2-6 years with both antiandrogens; the mechanism is unexplained. These observations open possibilities for new strategies in the use of antiandrogens.

Other side-effects of endocrine treatment are also timedependent and may not become relevant with the short treatment periods usually necessary for the management of metastatic disease. However, they may be very relevant for prolonged periods of endocrine treatment, such

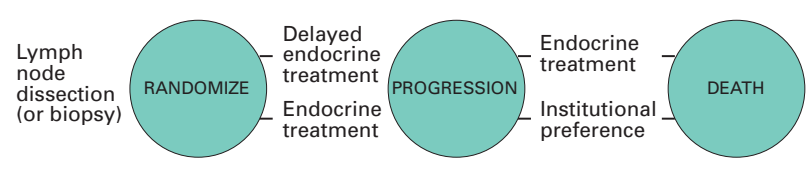

Fig. 3. The randomization scheme of the EORTC protocol 30846 , early vs delayed endocrine treatment in patients with pN1-3 prostate cancer.

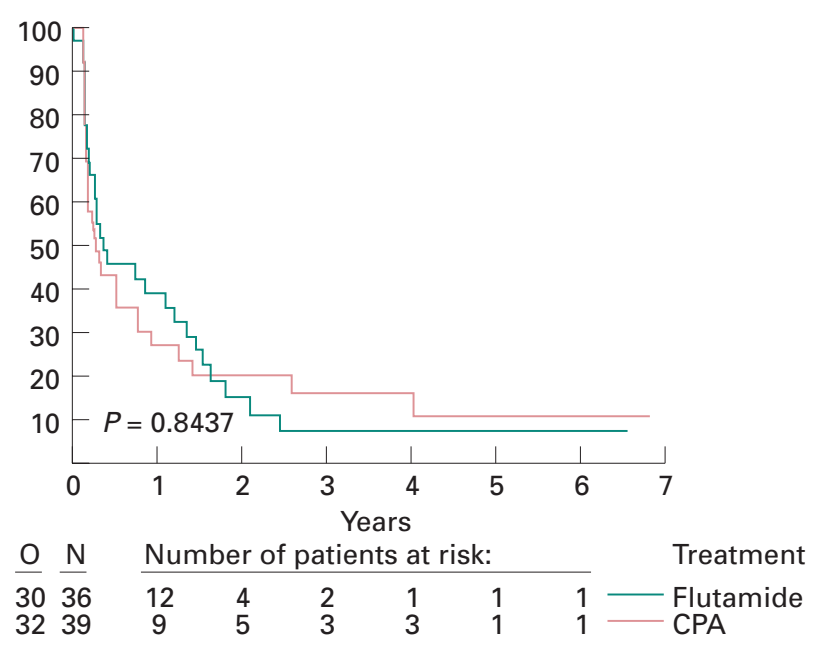

Fig. 4. The time to loss of ejaculation in 75 previously potent patients with metastatic prostate cancer treated by either flutamide (green) or cyproterone acetate (red) in a randomized study (EORTC protocol 30892).

as described above. Daniell [30] described an important increase in osteoporosis and osteoporotic fractures after orchidectomy in comparison with an age-matched population of men with intact testicular function, over a period of 9 years; the results are depicted in Fig. 5. The occurrence of a decrease in bone mineral density, which leads to osteoporotic fractures if critical limits are passed, is clearly related to the decrease of circulating androgens. This is also confirmed by Eriksson et al. [31], and McGrath and Diamond [32].

The pale appearance of many patients under endocrine treatment is caused by significant anaemia that results from low androgen levels. This has been quantified recently by Strum et al. [33]. A probable mechanism and the immediate recovery of red blood cell counts with androgen substitution have been described by Weber et al. [34].

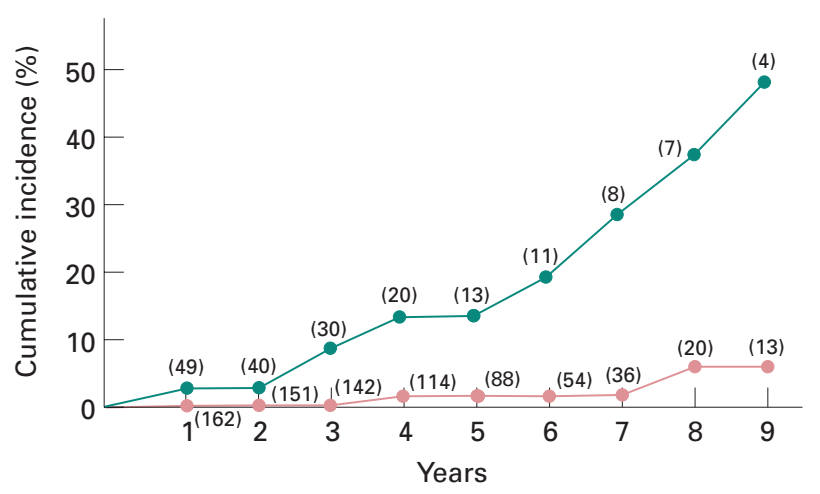

Fig. 5. The cumulative incidence of first osteoporosis fractures in men with prostate cancer with (green) and without (red) orchidectomy. (From Daniell [30] with permission). 
Low circulating testosterone levels also lead to muscular atrophy; this may be related to mechanisms described for the syndrome of spinal and bulbar muscular atrophy (SBMA), where normal androgen action at the level of the androgen receptor in skeletal musculature is no longer possible due to a mutation [35]. Obviously, loss of libido, anaemia and a decrease of muscle mass can easily explain the tiredness and apathy often seen under endocrine treatment.

Male androgen levels have been shown to decrease with age. This normal 'male climacteric' is different from the 'low androgen syndrome', which is the result of endocrine management of prostate cancer. A complete summary of the spectrum of symptoms of the "low testosterone syndrome' is given by Morley et al. [36].

\section{Future developments}

Obviously, the future is unknown, but in the case of endocrine treatment, several future developments are almost predictable. MAB, while useful in certain situations, offers no clinically relevant advantages in survival. The anticipated very long periods of endocrine treatment, if early treatment or adjuvant treatment is chosen or deemed necessary, will necessitate a stronger consideration of side-effects and quality of life than has been the case to date. Future clinical research must concentrate on such options. Intermittent endocrine treatment has been promoted by Goldenberg and Bruchovsky [37]. Large randomized studies are underway to further explore the value of this type of management in comparison with standard forms of treatment. Another concept, which has so far not been addressed in clinical studies, is the possibility of 'step-up treatment'. With early signs of clinical progression one could consider initiating endocrine treatment with the a $5 \alpha$-reductase inhibitor of the finasteride type. Subsequently, an antiandrogen could be used which, as shown here, may preserve sexual function, at least for some time. This could even be used intermittently to guarantee continuous sexual functioning. Once symptomatic progression occurs, more invasive principles of endocrine treatment could be applied. 'Step-up endocrine therapy' moves from minimally invasive to more invasive forms of endocrine management. Such regimens would have to be compared with standard forms of treatment to become clinically acceptable. Another important issue is whether there might be a justifiable compromise between quality of life and duration of life under endocrine treatment. This is a completely unexplored field. The issues of adjuvant endocrine treatment, intermittent treatment and endocrine prevention will be discussed in Part 2.

\section{References}

1 Schröder FH. Endocrine treatment of prostate cancer. In Walsh PC, Retik AB, Vaughan ED, Wein AJ, eds, Campbell's Urology. Chap 89. Philadelphia: WB Saunders Co., 1997: 2627-44

2 Robinson MRG, Smith PH, Richards B, Newling DWW, de Pauw M, Sylvester R. The final analysis of the EORTC Genito-Urinary Group Phase III clinical trial (Protocol 30805 ) comparing orchidectomy, orchidectomy plus cyproterone acetate and low dose stilboestrol in the management of metastatic carcinoma of the prostate. Eur Urol 1995; 28: 273-83

3 Labrie F, Dupont A, Belanger A et al. New approaches in the treatment of prostate cancer: complete instead of partial withdrawal of androgens. The Prostate 1983; 4: 579-94

4 Crawford ED, Eisenberger MA, McLeod Spaulding JT et al. A controlled trial of Leuprolide with and without Flutamide in prostatic carcinoma. New Eng J Med 1989; 321: 419-24

5 Denis LJ, Carneiro de Moura JL, Bono A et al. Goserelin acetate and flutamide versus bilateral orchiectomy: a phase III EORTC trial (30853). Urology 1993; 42: 119-30

6 LeLorier J, Grégoire G, Benhaddad A, Lapierre J, Derderian F. Discrepancies between meta-analyses and subsequent large randomized, controlled trials. New Engl J Med 1997; 337: 536-42

7 Prostate Cancer Trialists' Collaborative Group (PCTCG). Maximum androgen blockade in advanced prostate cancer: an overview of 22 randomised trials with 3283 deaths in 5710 patients. Lancet 1995; 346: 265-9

8 Prostate Cancer Trialists' Collaborative Group (PCTCG). Maximum androgen blockade in advanced prostate cancer: a metaanalysis of 22 randomised trials. Eur Urol Update Series 1995; 4: 186-94

9 Eisenberger MA, Blumenstein BA, Crawford ED et al. Bilateral orchiectomy with or without flutamide for metastatic prostate cancer. N Engl J Med 1998; 339: 1036-42

10 The Medical Research Council Prostate Cancer Working Party Investigators Group. Immediate versus deferred treatment for advanced prostatic cancer: initial results of the Medical Research Council Trial. Br J Urol 1997; 79: 235-46

11 de Voogt HJ, Studer U, Schröder FH et al. Final analysis of EORTC GU group trial 30843. Eur Urol 1998; 33: 152-8

12 van den Ouden D, Tribukait B, Blom JHM et al. Deoxyribonucleic acid ploidy of core biopsies and metastatic lymph nodes of prostate cancer patients: impact on time to progression. J Urol 1993; 150: 400-6

13 Byar DP, Corle DK. Hormone therapy for prostate cancer: results of the Veterans Administration Cooperative Urological Research Group Studies. NCI Monograph 1988; 7: $165-70$

14 Adolfsson J. Deferred treatment of low grade stage T3 prostate cancer without distant metastases. J Urol 1993; 149: 326-9

15 Pound CR, Partin AW, Walsh PC. The natural history of progression to metastases and death due to prostate cancer in men with PSA recurrence following radical prostatectomy. J Urol (Suppl) 1998; 159: 334, A1283 
16 van den Ouden D, Hop WCJ, Kranse R, Schröder FH. Tumour control according to pathological variables in patients treated by radical prostatectomy for clinically localized carcinoma of the prostate. Br J Urol 1997; 79: $203-11$

17 Johansson JE, Holmberg L, Johansson S, Bergström R, Adami HO. Fifteen-year survival in prostate cancer. A prospective, population-based study in Sweden. JAMA 1997; 277: 467-71

18 Adolfsson J, Steineck G, Hedlund P-O. Deferred treatment of clinically localized low-grade prostate cancer: actual 10-year and projected 15-year follow-up of the Karolinska series. Urology 1997; 50: 722-6

19 Lowe BA, Liström MB. Incidental carcinoma of the prostate: an analysis of the predictors of progression. J Urol 1988; 140: 1340-4

20 Epstein JI, Paull G, Eggleston JC, Walsh PC. Prognosis of untreated stage A1 prostatic carcinoma: a study of 94 cases with extended follow-up. J Urol 1986; 136: 837-9

21 Blute ML, Zincke H, Farrow GM. Long-term follow-up of young patients with stage A adenocarcinoma of the prostate. J Urol 1986; 136: 840-3

22 Stenman UH, Hakama M, Knekt P, Aromaa A, Teppo L, Leinonen J. Serum concentrations of prostate-specific antigen and its complex with $\alpha 1$-antichymotrypsin before diagnosis of prostate cancer. Lancet 1994; 334: 1594-8

23 Smart ChR. Prostate cancer facts and fiction. Guest Editorial. J Surg Oncol 1997; 66: 223-9

24 Newcomer LM, Stanford JL, Blumenstein BA, Brawer MK. Temporal trends in rates of prostate cancer: declining incidence of advanced stage disease, 1974-94. J Urol 1997; 158: 1427-30

25 Rietbergen J, Hoedemaeker RF. The changing pattern of prostate cancer at diagnosis. In Proceedings ESU Organized Symposium on 'Prostate Cancer - Epidemiology, Prevention and Endocrine Treatment', Budapest, Hungary, October 1997. New York, London: Parthenon Publishing Group, 1998: $10-2$

26 Blackard CE, Byar DP, Jordan WP. Orchiectomy for advanced prostatic carcinoma. Urology 1973; 1: 553-60

27 Andriole G, Lieber M, Smith J et al. Treatment with Finasteride following radical prostatectomy for prostate cancer. Urology 1995; 45: 491-7

28 Ellis WJ, Grayhack JT. Sexual function in aging males after orchiectomy and estrogen therapy. J Urol 1963; 89: 895-9

29 Schröder FH, and members of the EORTC Genitourinary Group. Prostate cancer treated by antiandrogens - is preservation of sexual function possible? 1998; in press

30 Daniell HW. Osteoporosis after orchiectomy for prostate cancer. J Urol 1997; 157: 439-44

31 Eriksson S, Eriksson A, Stege R, Carlström K. Bone mineral density in patients with prostatic cancer treated with orchidectomy and with estrogens. Calcif Tissue Int 1995; 57: 97-9

32 McGrath SA, Diamond T. Osteoporosis as a complication of orchiectomy in 2 elderly men with prostatic cancer. J Urol 1995; 154: 535-6

33 Strum SB, McDermed JE, Scholz MC, Johnson H, Tisman G. Anaemia associated with androgen deprivation in patients with prostate cancer receiving combined hormone blockade. Br J Urol 1997; 79: 933-41

34 Weber JP, Walsh PC, Peters CA, Spivak JL. Effect of reversible androgen deprivation on hemoglobin and serum immunoreactive erythropoietin in men. Am J Hematol 1991; 36: 190-4

35 Warner CL, Griffin JE, Wilson JD et al. X-linked spinomuscular atrophy: a kindred with associated abnormal androgen receptor binding. Neurology 1992; 42: 2181-4

36 Morley JE, Kaiser FE, Hajjar R, Perr HM III. Testosterone and frailty. Clin Geriat Med 1997; 13: 685-95

37 Goldenberg SL, Bruchovsky N, Gleave ME, Sullivan LD, Akakura K. Intermittent androgen suppression in the treatment of prostate cancer: a preliminary report. Urology 1995; 45: 839-45

\section{Author}

F.H. Schröder, MD, FEBU, Professor and Chairman, Department of Urology, Erasmus University and Academic Hospital Rotterdam, PO Box 1738, 3000 DR Rotterdam, The Netherlands.

\section{MCQs}

1 Androgens are produced in:

(a) the testis

(b) the testis and subcutaneous tissue

(c) the testis and adrenal glands

(d) the testis, adrenal glands, and subcutaneous tissues

2 Maximal androgen blockade:

(a) is the treatment of choice for prostate cancer.

(b) has been proven to be (more) effective.

(c) produces an advantage in 5-year survival of $5-10 \%$ above castration.

(d) is indicated for flare prevention, rapid induction of a response, and several other situations.

3 Endocrine treatment of prostate cancer:

(a) prolongs time to progression

(b) prolongs disease-specific survival

(c) was shown in randomized studies to prolong survival

(d) was shown to be superior to placebo in the VACURG studies

4 Median times to progression:

(a) are roughly halved by endocrine management

(b) depend strongly on stage and grade

(c) are an accurate measure of survival

(d) are an important endpoint of clinical studies

5 Endocrine treatment of prostate cancer (a) should routinely be given with biochemical evidence of progression 
(b) was shown beyond doubt to be more effective if applied early

(c) was shown beyond doubt to be equally effective with delayed application (VACURG studies)

(d) the timing is subject to patient choice and preference

6 Quality of life:

(a) is better with early endocrine treatment

(b) was shown to be improved with delayed treatment because patients remain potent

(c) is likely to depend on the duration of treatment

(d) has never been studied in patients with prostate cancer

7 Libido and potency:

(a) are lost very slowly with antiandrogen treatment

(b) sexual function is generally preserved under treatment with flutamide

(c) are always lost after castration

(d) are important elements of quality of life for all men with prostate cancer

8 Side-effects of endocrine treatment: (a) are not very relevant considering the seriousness of the disease

(b) depend strongly on the duration of treatment

(c) usually occur only after prolonged treatment

(d) are identical to symptoms of the male climacterium

9 Intermittent endocrine treatment

(a) is the treatment of choice in all men who wish to remain potent

(b) has been shown to be comparable to standard treatment for survival

(c) is currently the subject of phase III studies

(d) may be an option for men who do not show a favourable initial PSA response

10 Neo-adjuvant endocrine treatment:

(a) is probably effective before radiotherapy in T3 disease

(b) leads to a decrease of positive margins in $\mathrm{T} 3$ cases undergoing radiotherapy

(c) is usually given for 6 months

(d) leads to a significant advantage in time to progression if given for 6 months before radical prostatectomy 